\title{
Electrically Conducting Paper from a Polyaniline/Pulp Composite and Paper Folding Art Work for 3-D Object
}

\author{
Hiromasa Goto \\ University of Tsukuba, Japan
}

Corresponding author: H. Goto, University of Tsukuba, Tsukuba, Ibaraki, 305-8573, Japan. Email: gotoh@ims.tsukuba.ac.jp

Conducting polymer polyaniline (PANI) was synthesized in the presence of dispersed pulp fibers in the polymerization reaction to yield PANI/pulp composite fibers, which is then formed into a conducting paper sheet. The paper surface is observed using scanning electron microscopy, and electrical conductivity and electron spin resonance measurements are performed. This simple, convenient method for preparing organic conducting materials should prove useful for industrial applications, and the present results encourage further study in the new field of "textile electronics".

Keywords composite; conducting polymers; pulp; SEM

Polyaniline (PANI) is one of the most promising conducting polymers. The synthesis of PANI is different from the more general methods used for the production of other conducting polymers. For instance, polyacetylenes [1], polythiophenes [2], polypyrroles [3], and polyphenylene-vinylenes [4] are synthesized in organic media with the aid of metal complex catalysts under an argon or dry air atmosphere. In contrast, PANI is generally synthesized in water with an oxidizer [5-8]. Furthermore, production of PANI is possible in ambient atmosphere (without requiring inert gas).

PANI has been applied in various industrial applications such as a buffer layer in organic electroluminescent devices, organic electrodes, and conductors, because of its low cost, simplicity, and convenient synthesis. On the other hand, PANI is difficult to process. Although organic solvents, such an $N$-methylpyrrolidone and $m$-cresol, can dissolve PANI, the high boiling points and toxicity of these solvents are a barrier to the use of PANI films in industrial applications. To improve processability, an anionic surfactant has been employed in the synthesis of colloidal anilines [9].

Cellulose has been applied for industrial materials and contributed to enrichment of our life. Cellulose science and technology are progressing, such as application of 
ultrasound [10], wound-care materials for medical application [11]. Especially, cellulose nanofiber has been developed [12-14].

Many examples of conducting polymer composites have been prepared since discovery of conducting polymers [15]. Recently, nanocomposites from cellulose nanofibrils and PANI were developed [16].

Application of textile technology to the production of conducting materials is expected to lessen the processing drawbacks and new functionality [17]. PANI composite has been applied for actuators [18] and sensors [19]. In this study, PANI and pulp were combined. A PANI/pulp composite was successfully prepared by the polymerization of aniline monomers on the surface of pulp in water. The composite thus synthesized was formed into a conducting paper sheet, which had favorable electrical conductivity, and pulp-like processability.

An electrically conducting sheet was prepared, its surface structure was examined, and the charge carriers were examined using electron magnetic spin resonance (ESR).

\section{EXPERIMENTAL}

Preparation of PANI/pulp composite

Aniline, pulp, and $\mathrm{H}_{2} \mathrm{SO}_{4}$ were added to $60 \mathrm{~mL}$ of water in an Erlenmeyer flask at room temperature to form aniline sulfate. The mixture was stirred for $30 \mathrm{~min}$ at $0{ }^{\circ} \mathrm{C}$. Subsequently, $20 \mathrm{~mL}$ of pre-cooled ammonium persulfate (APS, $\left(\mathrm{NH}_{4}\right)_{2} \mathrm{~S}_{2} \mathrm{O}_{8}$ ) in water solution was slowly added to the mixture at $0{ }^{\circ} \mathrm{C}$. After $24 \mathrm{~h}$, the mixture was filtered, soaked, and vigorously washed in a large volume of water with magnetic stirrer for $12 \mathrm{~h}$, filtered again, and then washed in a large volume of methanol for $12 \mathrm{~h}$. After filtration, the PANI/pulp was dried. The quantities of aniline, $\mathrm{H}_{2} \mathrm{SO}_{4}$, APS, and water used in the experiments are summarized in Table 1. Twelve variations of aniline/pulp composite preparation were performed. The molecular structure of APS is shown in Figure 1(a).

Figure 1.

PANI was successfully deposited on the pulp surface. Usually, PANI residue attaches to the wall of the reaction vessel used in the polymerization. This wall residue is often difficult to remove. Interestingly, in the current work, the Erlenmeyer flask wall remained relatively clear. This may be due to the capillary effect of the pulp effectively causing both adsorption of the monomer between pulp fibers and absorption of monomer in individual pulp fibers, as shown in Figures 1(b) and (c). Subsequently, the polymerization reaction occurred on the surface of the pulp. The brushed pulp fibers 
functioned as a reaction field, yielding PANI. As a second alternative explanation for the visually clear flask wall, PANI residue on the wall of the reaction vessel may have been mechanically removed and/or washed off by the pulp and PANI/pulp during the reaction. This process allowed effective polymerization on the pulp to yield PANI-coated pulp (Figure 1(d)). A plausible cross-sectional structure of the fiber is shown in Figure 1(e). The skin of the fiber was coated with PANI, and the PANI layer then grew into the pulp.

\section{Papermaking}

A PANI/pulp composite sheet was prepared using a papermaking technique. Once the composite fibers were dispersed in a large volume of water, the suspension was filtered with a fine metal net. A wet sheet appeared on the filter net. The remaining water in the wet sheet was removed by pressing under a dry paper filter, and the sheet was then dried to yield a PANI/pulp composite paper. Figure 2(a) shows an image of the paper. The composite paper had a smooth surface and was sufficiently flexible to enable the preparation of paper-folding artwork ("origami"). Figures 2(b) and (c) show a conducting paper rose and a crane made from the PANI/pulp composite sheet, respectively.

A two-layer paper can also be prepared by the papermaking technique, as shown in Figure 2(d). First, a PANI/pulp composite sheet was prepared on the metal net using the above technique. Residual water in the composite was removed by pressing under a dry filter paper, and the sheet was peeled from the mesh. Next, a pure pulp sheet was fabricated on the net (without removing the residual water), and the composite was carefully placed on the wet paper. The stacked sheets were pressed under a dry paper filter to remove the water, and then dried to obtain a double-layer paper. The adhesion between the layers was very good. A dark emerald-green composite could be decollated by covering (or sandwiched) with another dyed paper (non conducting paper) layer by this method. Although the plain paper surface layer had no electrical conductivity, the double layer paper may serve as an electromagnetic barrier.

Furthermore, thick composite materials can be prepared in the same manner, as shown in Figure 2(e). Generally, PANI film is prepared by casting (evaporation of solvent from a polymer solution to form a solid film) from $\mathrm{N}$-methylpyrrolidone. Preparation of thick PANI films or ingots is very difficult by casting. In contrast, the papermaking technique easily allows thick sheets and ingots of organic conducting materials.

Figure 2. 


\section{RESULTS AND DISCUSSION}

\section{SEM}

Figure 3 displays scanning electron microscopic (SEM) images of the PANI/pulp composite. The lower magnification image indicates that the PANI coated the entire sheet, as shown in Figure 3(a,b). The higher magnification image shows fine fungus-like structures distributed over individual fibers (Figure 3(c.d)).

It has been reported that polyanilines show nanotube or nanofiber structures [20]. However, the polyaniline prepared in the presence of pulp (cellulose) shows neither tubes nor fibers. The characteristic fungus-like structure of the composite in the present study can be derived from self-aggregation of the main chain. Interaction between hydroxy group in the cellulose and self-aggregation properties of the polyaniline during the polymerization allows formation of the sphere structure.

Figure 3.

\section{ESR}

The PANI thus synthesized in the composite was in an oxidized (doped) state. It had charge carriers (radical cations and dications), and displayed electrical conductivity. ESR measurements were used to detect radicals in the PANI charge carriers. The ESR spectra of PANI/pulp composites are shown in Figure 4. The line shapes of the ESR signal are asymmetric Dysonian pattern observable for conducting polymers. The signal intensity from the samples increased with aniline feed ratio (corresponding to the amount of PANI in the composite) in the polymerization. This is because of an increase in charge carriers with increasing PANI content in the pulp fibers. Thus, a high PANI content in the composite afforded a high concentration of radicals.

Figure 4.

\section{Electrical conductivity}

The electrical conductivity of the PANI/pulp composite was examined by the four-probe method. The data are summarized in Table 1. The conductivity was somewhat low $\left(<10^{7} \mathrm{~S} / \mathrm{cm}\right.$ ) at low PANI/pulp ratios ([aniline feed amount in the polymerization]/[pulp]). The conductivity increased to $1.9 \times 10^{2} \mathrm{~S} / \mathrm{cm}$ with increasing polymer ratio, as shown in Figure 5. The conductivity became saturated at a ratio of about $35 \mathrm{wt} \%$. Although conductivity of the composite is low compared to that of the 
PANI nanofiber [22], a composite with low PANI content and moderate conductivity (< $10^{2} \mathrm{~S} / \mathrm{cm}$ ) could be useful for applications for industrial use such as anti-static envelopes, conducting packages, and corrosion inhibitors.

\section{Mechanical properties}

The mechanical properties of the PANI/pulp paper sheet were examined by constant rate elongation method. Figure 6 shows stress curves as a function of elongation for the $\mathrm{PANI} /$ pulp paper (sample $=$ run 12 in Table 1 ) and the standard paper at a rate of 20 $\mathrm{mm} / \mathrm{min}$ at $23{ }^{\circ} \mathrm{C}$. The PANI/pulp displays lower tensile strength $(P)(P$ PANI/pulp $=1.5$ $\left.\mathrm{N} / \mathrm{mm}^{2}\right)$ than that of the standard paper $\left(P_{\text {pulp }}=2.6 \mathrm{~N} / \mathrm{mm}^{2}\right)$. Gradually, fracture of the standard paper and the PANI/pulp paper sheet proceeded between $1.49 \mathrm{~mm}$ and 2.60 $\mathrm{mm}$, and $0.93 \mathrm{~mm}$ and $1.60 \mathrm{~mm}$ in elongation, respectively, indicating the strength of the PANI/pulp is lower than that of the standard paper. These results can be due to the fact that the hydrogen bonding between pulp fibers is depressed by the PANI coating layer. However, the functionality of the flexibility and the strength can be moderate for practical application as a conducting paper sheet.

Figure 6.

Figure 7 shows conductivity vs. twist angle for the composite sample. Here, the conductivity was calculated by the resistance $(\rho)$ of the sample. Conductivity of the composite is almost constant at low bend angles. The conductivity increases with the angle $(\theta)$ larger than $250^{\circ}$. This may be due to the fact that the sample is partly compressed by the twist process. The mechanical pressure makes dense and promotes electronic contact between individual fibers in the sheet, resulting in the increase of electrical conductivity. The strain sensitive function may be applied for strain sensors.

Figure 7.

\section{CONCLUSION}

$\mathrm{PANI} /$ pulp composite was prepared by polymerization in the presence of pulp. The composite fiber was processed using common papermaking techniques. The present research provides a new production method for conducting polymer sheets. This technique allows production of lower cost versions of anti-static flexible envelopes and conducting package. The paper folding technique (Origami) allows preparation of a three-dimensional object with conducting polymers [22]. 
This very simple and convenient method for preparing organic conducting materials should prove useful for industrial applications, and may contribute to new developments in the field of "textile electronics".

\section{Experimental}

Absorption spectra were obtained using a Hitachi U-2000 spectrophotometer. ESR measurements of solid samples of the polymer were carried out with a JEOL JES TE-200 spectrometer with $100 \mathrm{kHz}$ modulations. Electrical conductivities for the samples were obtained with Mitsubishi MCP-T610 and Hioki SM8213. The tension measurements were carried out with Autograph 100KN, AG-X (Shimazu, Japan).

\section{Materials}

Aniline (Tokyo Kasei, TCI) and water were distilled prior to use. APS (Kanto Chemicals, Japan) was used as received.

\section{Funding}

This work was supported by the ILC project of University of Tsukuba [H18, Goto project].

\section{Acknowledgements}

The author would like to thank Glass work shop of Central Workshop of University of Tsukuba for fine glass works, A. Goto for the paper-folding artwork, and A. Yokoo for assistance.

\section{REFERENCES}

1. Ito, T. and Shirakawa, H., Thermal Cis-Trans Isomerization and Decomposition of Polyacetylene. J. Polym. Sci. Polym. Chem. Ed., 13, 1943-1950 (1975).

2. Yamamoto, T., Sanechika, K. and Yamamoto, A., Preparation of Thermostable and Electric-Conducting Poly(2,5-thienylene). J. Polym. Sci. Polym. Lett. Ed., 18, 9-12 (1980).

3. Havinga, E. E., Horssen, L. W., L., Hoeve, W., Wynberg, H. and Meyer, E. W., Sefl-Doped Water-Soluble Conducting Polymers. Polym. Bull., 18, 277-281 (1987).

4.Schlüter, A. D. Synthesis of Poly(para-phenylene)s. In Handbook of conducting polymers, second edition, Skotheim, T. A., Elsenbaumer, R. L. and Reynolds, J. R. (Eds), pp. 209-224, Mercel Dekker, New York, 1998.

5. Andretta, A., Cao, Y., Chiang, J.-C., and Heeger, A. J., Electrically-Conductive 
Fibers of Polyaniline Spun from Solutions in Concentrated Sulfuric Acid. Synth. Met., 26, 383-389 (1988).

6. Cao, Y. and Heeger, A. J., Charged Solitons in Pernigraniline. Synth. Met., 39, 205-214 (1990).

7. MacDiarmid, A. G. and Epstein, A. J., The Concept of Secondary Doping as Applied to Polyaniline. Synth. Met., 65, 103-116 (1990).

8. Geniés, E. M., Boyle, A., Lapkowski, M. and Tsintavis C., Polyaniline: A Historical Survey. Synth. Met., 36, 139-182 (1990).

9. Su, S.-J. and Kuramoto, N., Synthesis of Processable Polyaniline Complexed with Anionic Surfactant and Its Conducting Blends in Aqueous and Organic System. Synth. Met., 108, 121-126 (2000).

10. Tomljenović, A. and Čunko, R., Reducing Fibrillation Tendency of Man-Made Cellulose Fibres Employing Ultrasound Treatment. J. Text. Inst., 95, 327-339 (2004).

11. Miraftab, M., Qiao, Q., Kennedy, J. F., Knill, C. J. and Groocock, M. R., Advanced Wound-Care Materials: Ultra High Absorbing Fibres Made from Alginates Containing Branan Ferulate and Carboxymethyl. J. Text. Inst., 95, 341-348 (2004).

12. Bhatnagar, A. and Sain, M., Processing of Cellulose Nanofiber-Reinforced Composites, Faculty of Forestry. J. Reinf. Plast. Comp., 24, 1259-1268 (2005).

13. Okawa, K., Hayashi, S., Nishida, A., Yamamoto, H. and Ducreux, J., Preparation of Pure Cellulose Nanofiber via Electrospinning. Textile Res. J., 79, 1396-1491 (2009).

14. Ma, Z., Kotak, M. and Ramakrishna, S., Electrospun Cellulose Nanofiber as Affinity Membrane. J. Membrane Sci., 265, 115-123 (2005).

15. Goto, H., Electroconducting Polymer Composite Paper and Its Production. Jpn Patent, JP 1998-342089 (Dec 1, 1998), Chem. Abstr. 133:19016.

16. Mattoso, L. H. C., Medeiros, E. S., Baker, D. A., Avloni, J., Wood, D. F. and Orts, W. J., Electrically Conductive Nanocomposites Made from Cellulose Nanofibrils and Polyaniline. J . Nanosci. Nanotech., 9, 2917-2922 (2009).

17. Volokhina, A.V., Use of Polyaniline for Fabrication of Promising Synthetic Fibres. Fibre Chem., 26, 9-16 (1994).

18. Yun, S., Kim, J., Multiwalled-Carbon Nanotubes and Polyaniline Coating on Electro-Active Paper for Bending Actuator. J. Phys D-Appl. Phys. 39, 2580-2586 (2006).

19. Dutta, D., Sarma, T. K., Chowdhury, D. and Chattopadhyay, A., A Polyaniline-Containing Filter Paper that Acts as a Sensor, Acid, Base, and 
Goto, H. Textile Research Journal 81(2) 122-127 (2011)

Endpoint Indicator and Also Filters Acids and Bases. J. Colloid. Interface Sci., 153-159 (2005).

20. Li, D., Huang, J. and Kaner, R. B., Polyaniline Nanofibers: A Unique Polymer Nanostructure for Versatile Applications. Acc. Chem. Res., 42, 135-145 (2009).

21. Abdelaziz, R., Duck Joo, T., Synthesis of Highly Conductive Polyaniline Nanofibers. Mater. Lett., 62, 4311-4314 (2008).

22. Goto, H. and Yokoo., A. Electroconducting Polymer Paper III. Fiber Prep. Jpn, 63, 114 (Jun 18, 2008). 
Goto, H. Textile Research Journal 81(2) 122-127 (2011)

Table 1 Quantities of compounds used for the oxidative polymerization in the presence of pulp for the PANI/pulp paper sheets, and conductivities.

\begin{tabular}{cclllll}
\hline Run $^{1}$ & $\begin{array}{c}\text { Monomer }^{2} \\
\text { vs. pulp } \\
\text { (wt \%) }\end{array}$ & $\begin{array}{c}\text { Monomer }^{2} \\
(\mathrm{~g})\end{array}$ & $\begin{array}{c}\mathrm{APS}^{4} \\
(\mathrm{~g})\end{array}$ & $\begin{array}{c}\mathrm{H}_{2} \mathrm{SO}_{4} \\
(\mathrm{~g})\end{array}$ & $\begin{array}{c}\text { Water } \\
(\mathrm{mL})\end{array}$ & $\begin{array}{c}\text { Conductivity }^{5} \\
(\mathrm{~S} / \mathrm{cm})\end{array}$ \\
\hline 1 & 2 & 0.06 & 0.06 & 0.02 & 80 & $<10^{-7}$ \\
2 & 4 & 0.12 & 0.12 & 0.04 & 80 & $<10^{-7}$ \\
3 & 8 & 0.24 & 0.24 & 0.08 & 80 & $<10^{-7}$ \\
4 & 10 & 0.3 & 0.3 & 0.1 & 80 & $6.2 \times 10^{-6}$ \\
5 & 15 & 0.45 & 0.45 & 0.15 & 80 & $9.9 \times 10^{-5}$ \\
6 & 20 & 0.6 & 0.6 & 0.2 & 80 & $3.6 \times 10^{-4}$ \\
7 & 25 & 0.75 & 0.75 & 0.25 & 80 & $1.1 \times 10^{-3}$ \\
8 & 30 & 0.9 & 0.9 & 0.3 & 80 & $5.7 \times 10^{-3}$ \\
9 & 35 & 1.05 & 1.05 & 0.35 & 80 & $1.1 \times 10^{-2}$ \\
10 & 40 & 1.2 & 1.2 & 0.4 & 80 & $1.2 \times 10^{-2}$ \\
11 & 45 & 1.35 & 1.35 & 0.45 & 80 & $1.7 \times 10^{-2}$ \\
12 & 50 & 1.5 & 1.5 & 0.5 & 80 & $1.9 \times 10^{-2}$ \\
\hline
\end{tabular}

${ }^{1}$ Solution 1: A solution of dispersed pulp, aniline, and $\mathrm{H}_{2} \mathrm{SO}_{4}$ in water $(60 \mathrm{~mL})$ was stirred for $30 \mathrm{~min}$ at $0^{\circ} \mathrm{C}$. Solution 2: APS in water $(20 \mathrm{~mL})$ at $0^{\circ} \mathrm{C}$. Solution 2 was added to solution 1 , and stirred for $24 \mathrm{~h}$ at $0^{\circ} \mathrm{C}$.

${ }^{2}$ Aniline

${ }^{3} \mathrm{NBKP} / \mathrm{LBKP}=70 / 30$, Canadian standard freeness $(\mathrm{CSF})=528 \mathrm{~mL}$

${ }^{4} \mathrm{APS}=$ ammoinium persulfate, $\left(\mathrm{NH}_{4}\right)_{2} \mathrm{~S}_{2} \mathrm{O}_{8}$.

${ }^{5}$ Measured using the four-probe method. 
Goto, H. Textile Research Journal 81(2) 122-127 (2011)

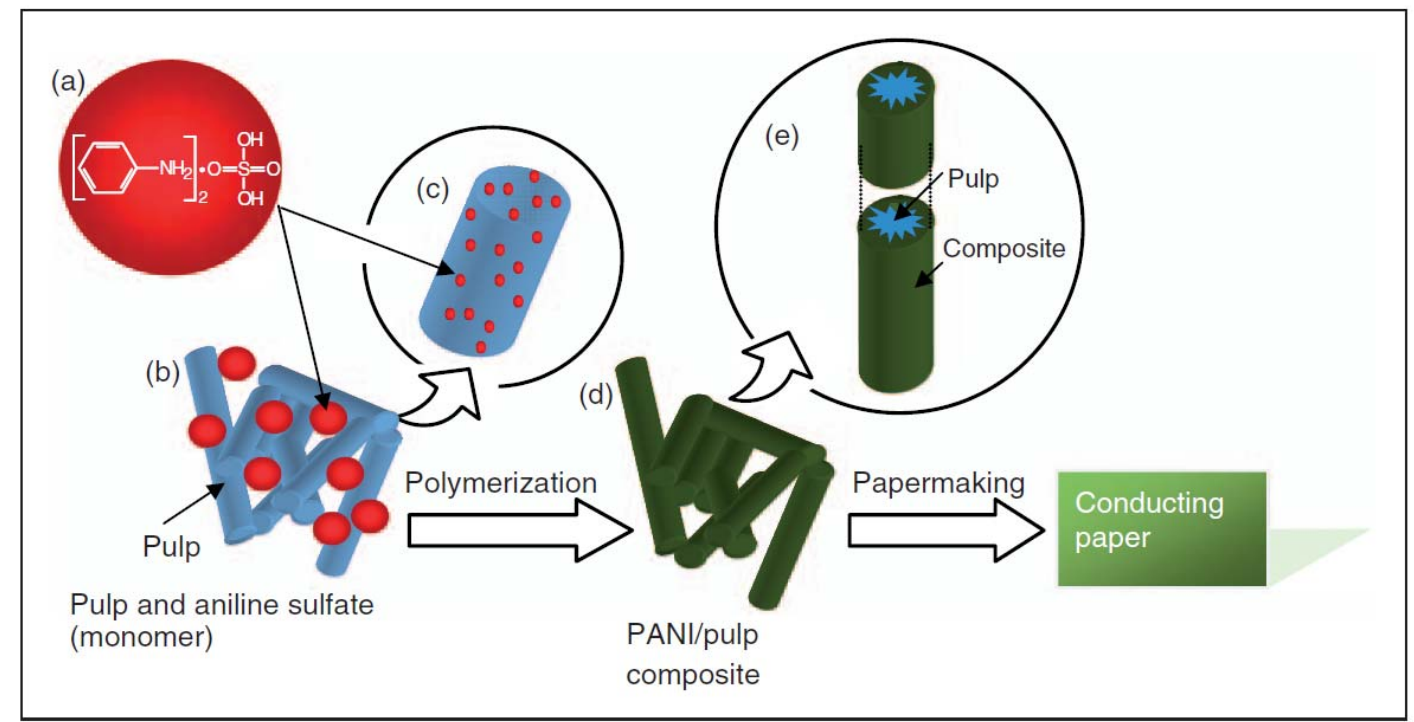

Figure 1 Schematic illustration of preparation of conducting paper sheet. (a) Aniline sulfate (monomer). (b) Monomers between individual pulp fibers. (c) Absorbed monomers inside the pulp fibers. (d) PANI/pulp composite. (e) Cross section of the composite. 
Goto, H. Textile Research Journal 81(2) 122-127 (2011)
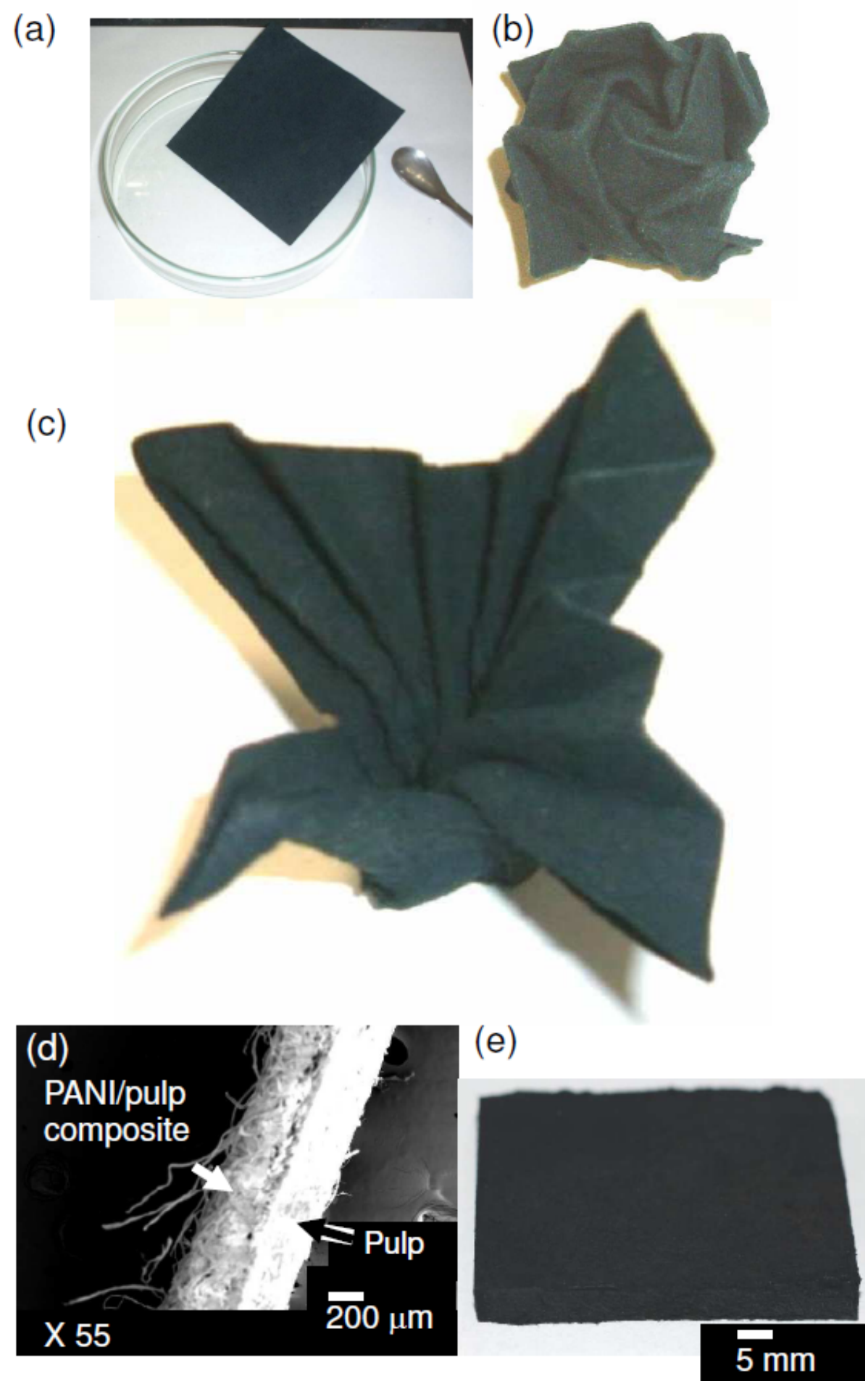

Figure 2 (a) Conducting paper sheet based on a PANI/pulp composite prepared by a paper processing technique. A conducting paper rose (b) and crane (c) made from a PANI/paper composite sheet. (d) Scanning electron microscopic (SEM) image of a cross-section of a double-layer sheet (paper/PANI and pulp composite). (e) Thick sample. 
Goto, H. Textile Research Journal 81(2) 122-127 (2011)

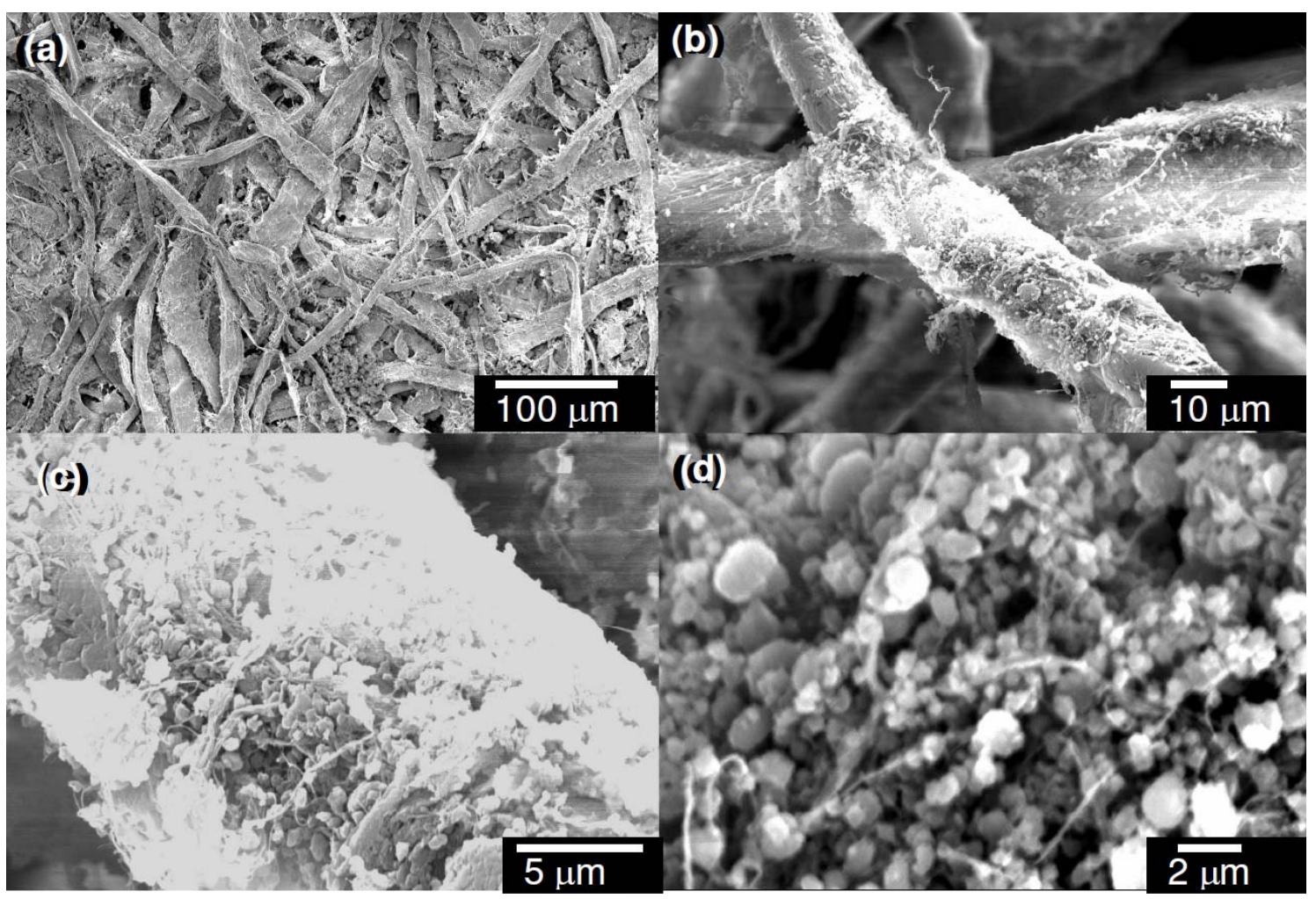

Figure 3 Surface images of PANI/pulp composite (sample = run 12 in Table 1) obtained using scanning electron microscopy (SEM). (a) $230 \mathrm{x}$, (b) $1200 \mathrm{x}$, (c) $5000 \mathrm{x}$, (d) $6000 \mathrm{x}$. 


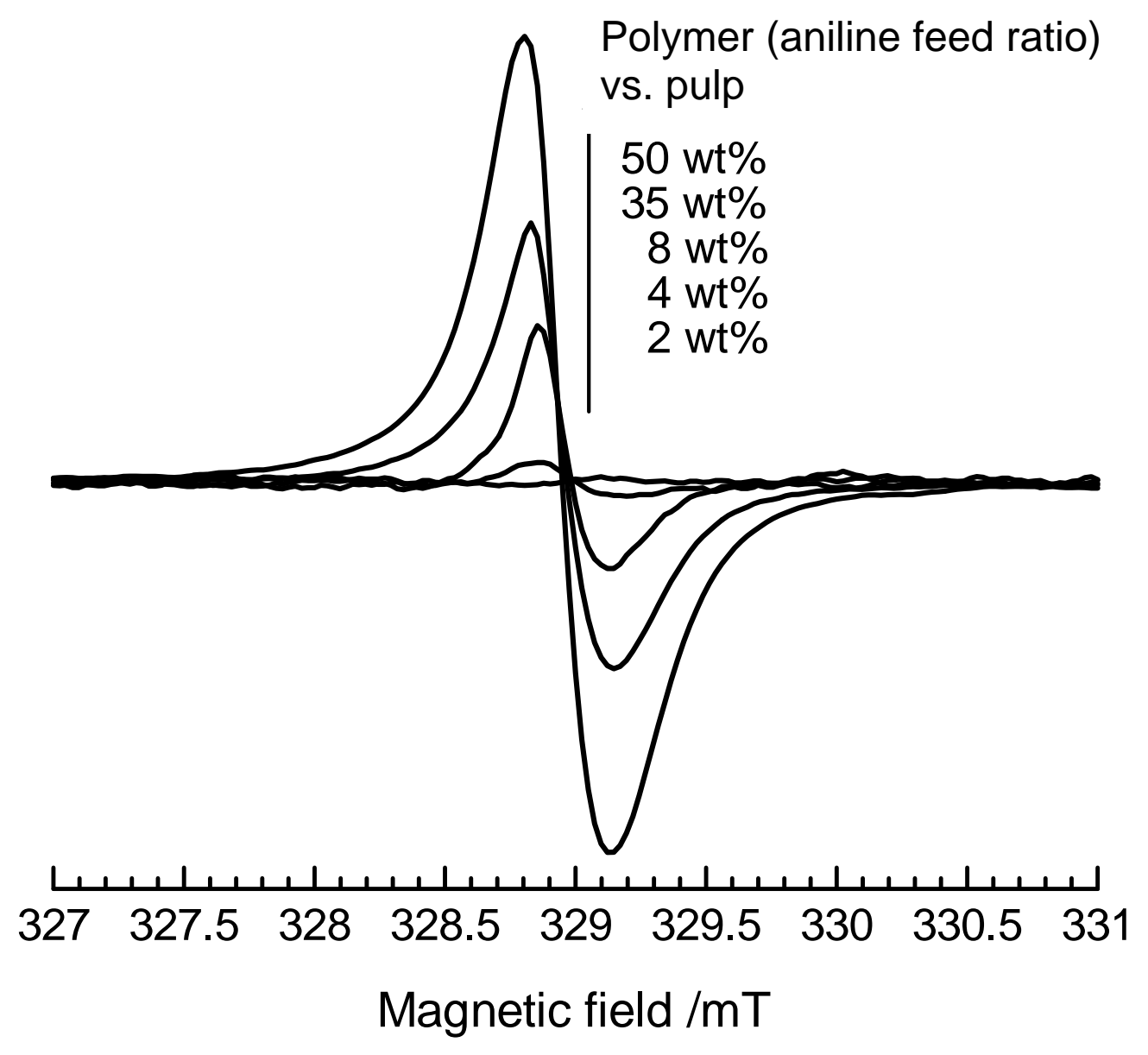

Figure $4 \mathrm{ESR}$ spectra of PANI/pulp composite. 
Goto, H. Textile Research Journal 81(2) 122-127 (2011)

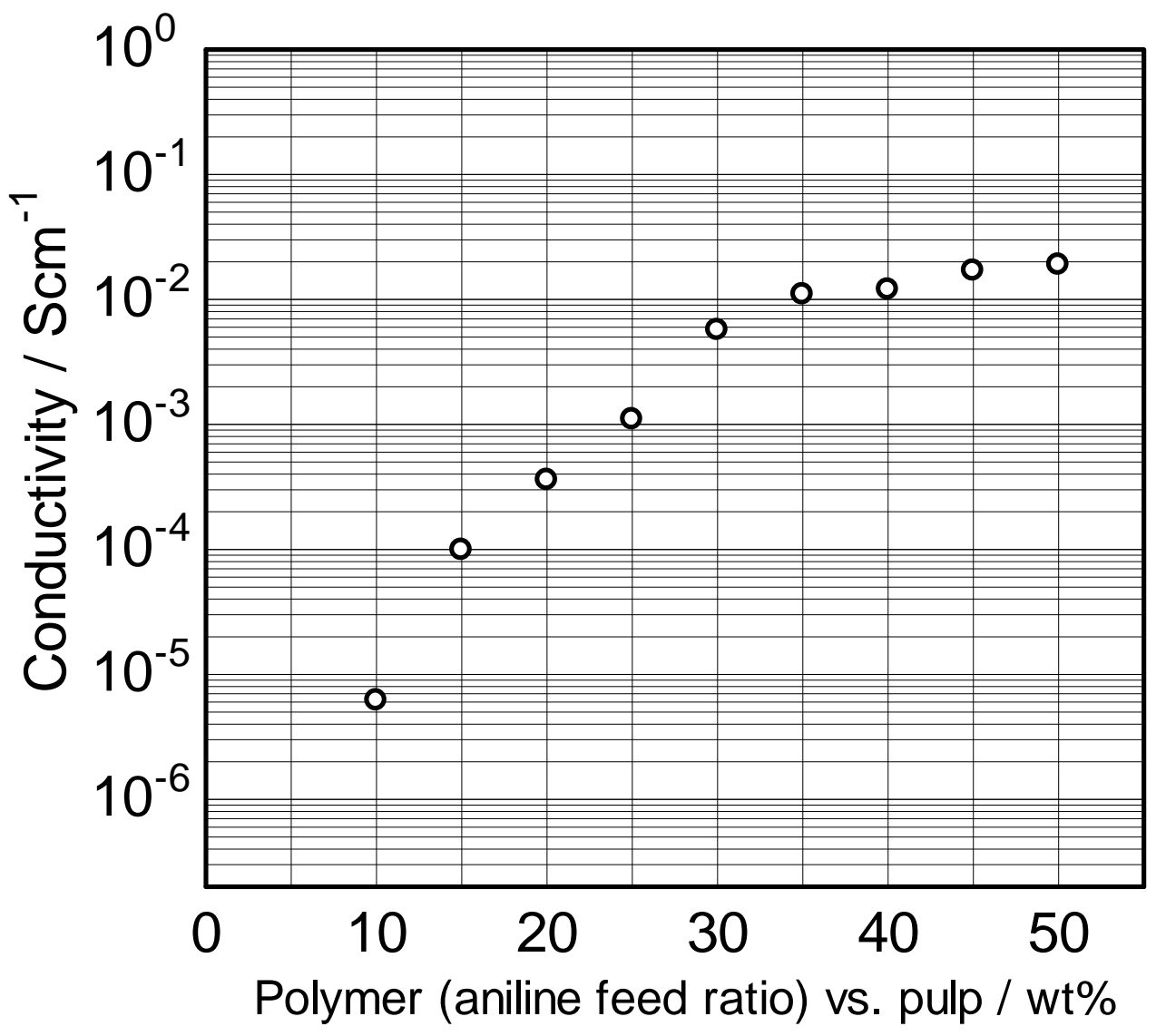

Figure 5 Electrical conductivity of PANI/pulp composite sheets. 
Goto, H. Textile Research Journal 81(2) 122-127 (2011)

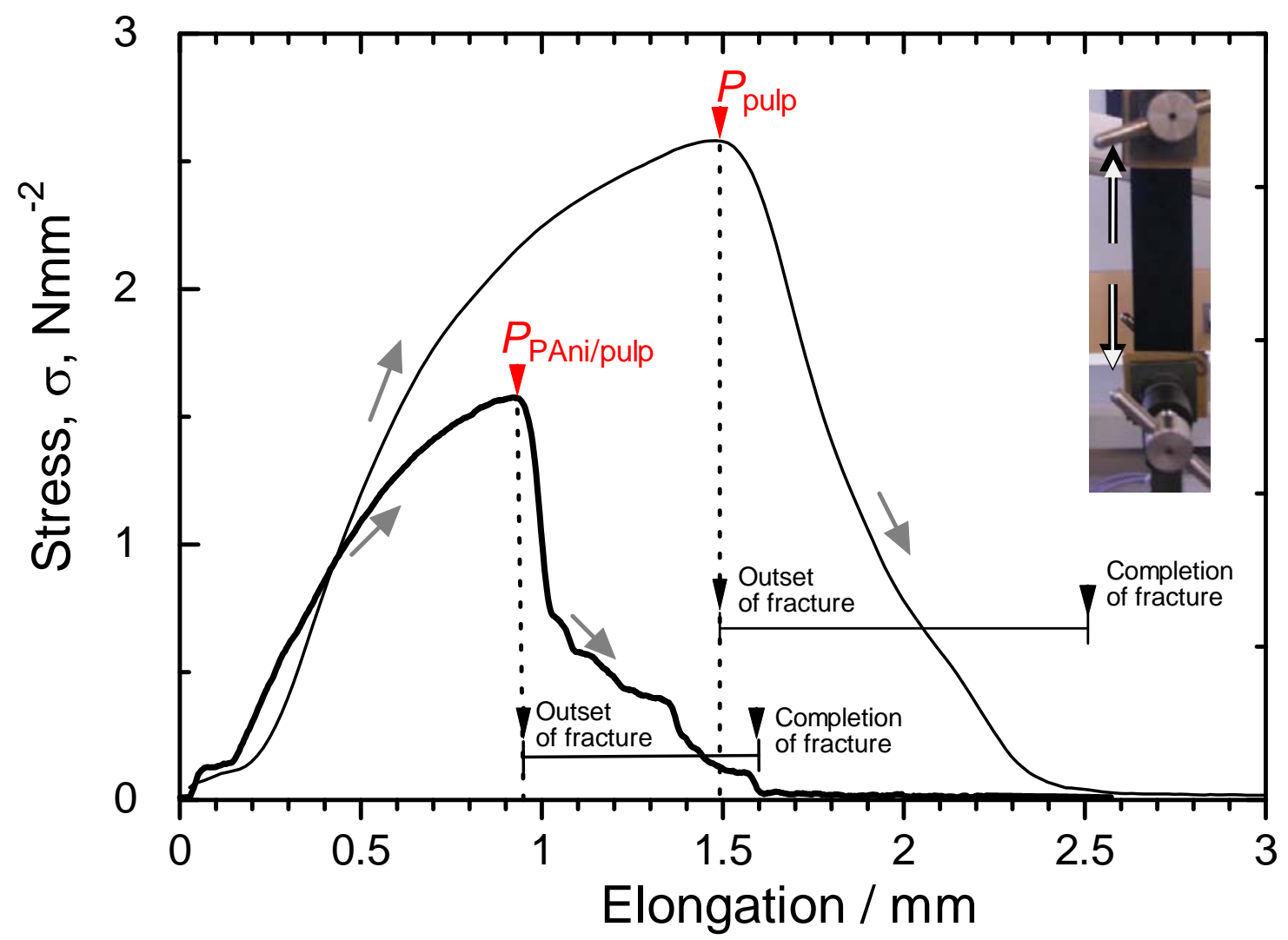

Figure 6 Stress $(\sigma)$ as a function of elongation in tensile measurements for the PANI/pulp paper sheet (thick line, sample = run 12 in Table 1) and a paper sheet without conducting polymer. $P=$ tensile strength. Elongation rate $=20 \mathrm{~mm} / \mathrm{min}$. Inset shows sample setting for the tensile strength measurement. 
Goto, H. Textile Research Journal 81(2) 122-127 (2011)

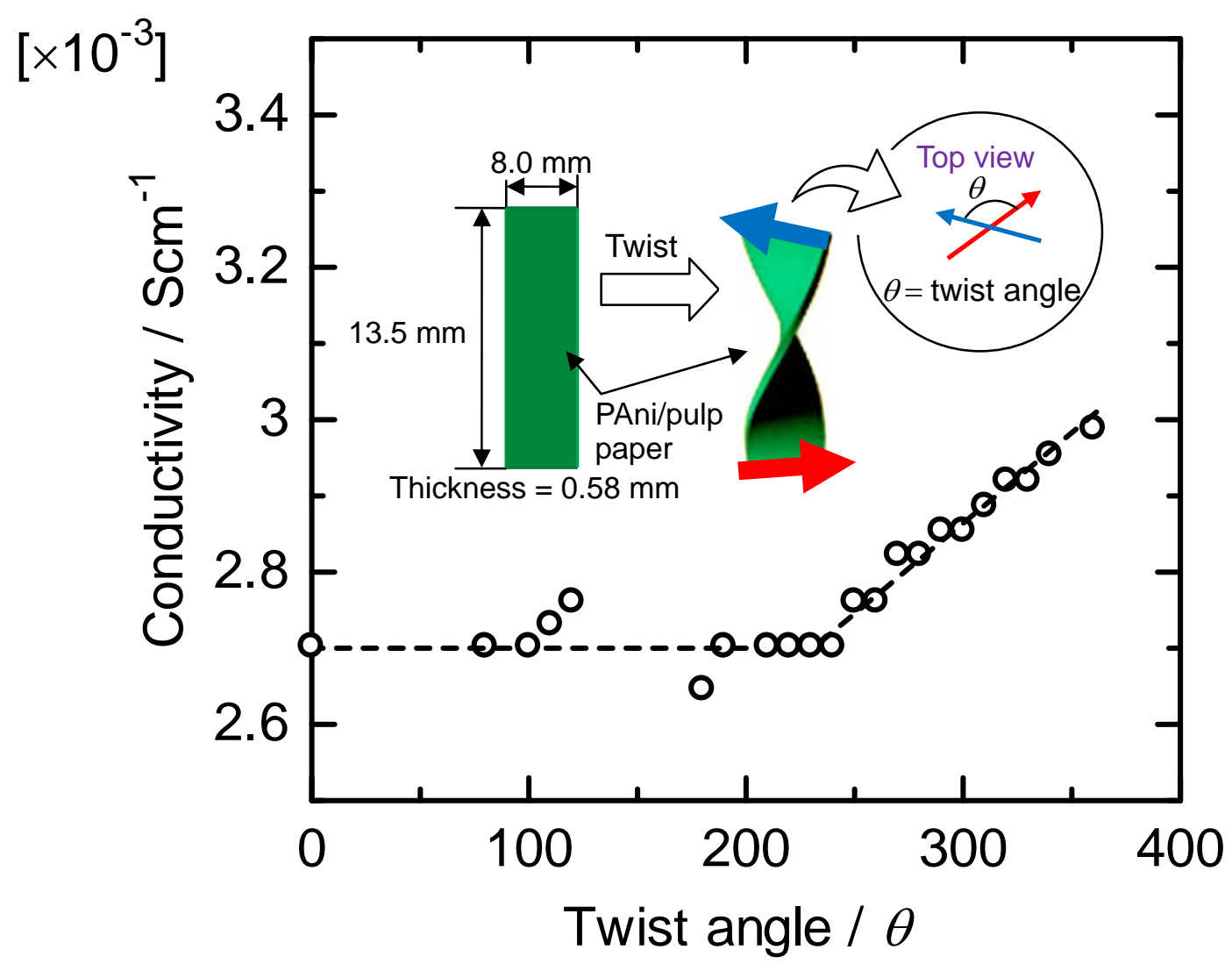

Figure 7 Conductivity of polyaniline/pulp paper sheet as a function of twist angle (degree, $\theta$ ). Inset shows sample size and twist of the PANI/pulp paper sheet. The conductivity values were estimated from the resistance values. 